\title{
MSI2 Gene
}

National Cancer Institute

\section{Source}

National Cancer Institute. MSI2 Gene. NCI Thesaurus. Code C91356.

This gene is involved in translational regulation. 